\title{
L-alanyl-L-glutamine ingestion maintains performance during a competitive basketball game
}

Jay R Hoffman*, David R Williams, Nadia S Emerson, Mattan W Hoffman, Adam J Wells, Daniele M McVeigh, William P McCormack, Gerald T Mangine, Adam M Gonzalez and Maren S Fragala

\begin{abstract}
Background: The purpose of this study was to examine the efficacy of L-alanyl-L-glutamine (AG) ingestion on basketball performance, including jump power, reaction time, shooting accuracy and fatigue.

Methods: Ten women (21.2 \pm 1.6 years; height: $177.8 \pm 8.7 \mathrm{~cm}$; body mass: $73.5 \pm 8.0 \mathrm{~kg})$, all scholarship NCAA Division I basketball players, volunteered for this study. Subjects participated in four trials, each consisting of a 40min basketball game with controlled time-outs for rehydration. During the first trial (DHY) subjects were not allowed to rehydrate, and the total weight lost during the contest was used to determine fluid replenishment during the subsequent three trials. During one trial subjects consumed only water (W), while during the other two trials subjects consumed the AG supplement mixed in water using either a low dose (1 g per $500 \mathrm{ml}$ ) (AG1) or high dose (2 g per $500 \mathrm{ml}$ ) (AG2) concentration. All data assessed prior to and following each game were converted into a $\Delta$ score (Post results - Pre results). All performance data were then analyzed using a one-way repeated measures analysis of variance.
\end{abstract}

Results: During DHY subjects lost $1.72 \pm 0.42 \mathrm{~kg}(2.3 \%)$ of their body mass. No differences in fluid intake (1.55 \pm $0.43 \mathrm{~L})$ were seen between rehydration trials. A $12.5 \%(p=0.016)$ difference in basketball shooting performance was noted between DHY and AG1 and an 11.1\% ( $p=0.029)$ difference was seen between AG1 and W. Visual reaction time was significantly greater following $A G 1(p=0.014)$ compared to DHY. Differences $(p=0.045)$ in fatigue, as determined by player loads, were seen only between AG2 and DHY. No differences were seen in peak or mean vertical jump power during any trial.

Conclusion: Rehydration with AG appears to maintain basketball skill performance and visual reaction time to a greater extent than water only.

Keywords: Supplement, Dehydration, Hypohydration, Exercise, Reaction Time

\section{Background}

Glutamine ingestion during acute dehydration stress is reported to enhance fluid and electrolyte absorption resulting from intestinal disorders [1-3], but it's effects may not be consistent [4]. This is possibly related to stability issues of glutamine in the gut. However, when glutamine is combined with alanine the ability to enhance electrolyte and fluid absorption appears to be greater than glutamine alone, likely via a combination of greater

\footnotetext{
* Correspondence: jay.hoffman@ucf.edu
Sport and Exercise Science, University of Central Florida, 4000 Central Florida

* Correspondence: jay.hoffman@ucf.edu Blvd, Orlando, Florida 32816, USA
}

(C) 2012 Hoffman et al; licensee BioMed Central Ltd. This is an Open Access article distributed under the terms of the Creative Commons Attribution License (http://creativecommons.org/licenses/by/2.0), which permits unrestricted use, distribution, and reproduction in any medium, provided the original work is properly cited. ion transporters within intestinal epithelia [5]. In addition, the greater stability of the alanine-glutamine dipeptide appears to be quite evident at a low $\mathrm{pH}[6]$. This could have important implications for athletes during competition.

Recently, acute ingestion of an alanine-glutamine dipeptide (AG) was reported to enhance fluid uptake and reduce the magnitude of performance decrement during exercise to exhaustion under hypohydrated conditions [7]. Furthermore, the alanine-glutamine dipeptide was shown to be significantly more effective than 
water alone. This has important implications during athletic performance, where dehydration can play a critical role in the outcome of a contest. For instance, a significant performance decrement has been shown with hypohydration levels of only $2 \%$ in basketball players $[8,9]$. This level of hypohydration has been shown to decrease field goal percentage in basketball players by $8 \%$, clearly affecting the potential outcome of a game. Considering that a thirst sensation may not appear until this level of hypohydration has already been reached [10], it becomes critical for athletes to rehydrate even when they do not feel the need to drink. Furthermore, rehydration does appear to be a major issue among basketball players. Nearly half of professional basketball players assessed prior to competitive games were found to be dehydrated prior to the onset of a basketball game, and that fluid intake during the games was not able to compensate for the pregame hypohydration [11]. In light of these findings, it appears that examining rehydration strategies in basketball players is warranted. Thus, the purpose of this study was to examine the efficacy of two different doses $(1 \mathrm{~g}$ per $500 \mathrm{ml}$ and $2 \mathrm{~g}$ per $500 \mathrm{ml}$ ) of AG on basketball performance, including jump power, reaction time, shooting ability and fatigue during a basketball game.

\section{Methods}

\section{Subjects}

Ten women volunteered for this study $(21.2 \pm 1.6$ years; height: $177.8 \pm 8.7 \mathrm{~cm}$; body mass: $73.5 \pm 8.0 \mathrm{~kg}$ ). Following an explanation of all procedures, risks, and benefits, each subject gave her informed consent to participate in this study. The Institutional Review Board of the University approved the research protocol. Subjects were not permitted to use any additional nutritional supplementation during the course of the study. Screening for additional supplement use was accomplished via a health history questionnaire completed during subject recruitment. All subjects were scholarship athletes playing for the University's Women's basketball team. The study protocol was a double-blind cross-over design.

\section{Testing protocol}

Data collection occurred on four separate occasions. Each session required subjects to participate in a 40-min basketball game (normal duration for a NCAA college basketball game). To simulate an actual competition, a 2-min time out was used at the 10-min mark of each half, and a 10-min halftime separated the first and second halves. Subjects were divided into two equally talented teams as determined by the team's player captains. The team members remained the same for each game. Thus level of competition (subjects competing against each other) was the same for each contest. Interestingly, each team won two games. The difference between each contest was the type of hydration fluid that was provided. During the first session (DHY) subjects were not allowed to rehydrate. During this session the total weight lost during the contest was determined, which was then used to determine the fluid replenishment during the subsequent three experimental sessions. During these three sessions subjects were provided fluid every $10 \mathrm{~min}$ in equal amounts for a total of six hydration times. The fluid consumed at each ingestion point was equal to the fluid loss observed during session one, divided by six. During one of these sessions subjects consumed only water (W), while during the other two session subjects consumed the AG supplement marketed as Sustamine ${ }^{\mathrm{TM}}$ (Kyowa Hakko USA, New York, NY) mixed in water using either a low dose (1 g per $500 \mathrm{ml}$ ) (AG1) or high dose (2 g per $500 \mathrm{ml}$ ) (AG2) concentration. The order of the three sessions was randomly determined per subject. All subjects were expected to begin each game in a euhydrated state. Prior to each contest a urine sample was analyzed for urine specific gravity $\left(\mathrm{U}_{\mathrm{sg}}\right)$ by refractometry to document euhydration; $U_{\mathrm{sg}} \leq 1.020$ was defined as euhydration [12]. If a subject's $U_{s g}>1.020$ she was requested to ingest $500 \mathrm{ml}$ of water and retested.

\section{Performance measurements}

Prior to each testing session all subjects performed a 10min dynamic warm-up. This warm-up was the same warm-up these athletes performed prior to every game during the competitive season. Following the warm-up subjects performed power, reaction and basketball shooting assessments. All testing sessions were supervised by certified strength and conditioning specialists. At the conclusion of the basketball game and final hydration intake, subjects performed all performance measures. Order of performance testing was performed in a randomized fashion for both pre-game and postgame assessments. Test-retest reliabilities for all assessments were $\mathrm{R}>0.90$.

\section{Power}

To quantify vertical jump power subjects performed five consecutive countermovement jumps (CMJ). During each jump subjects stood with their hands on their waist at all times. The subjects were instructed to maximize the height of each jump while minimizing the contact time with the ground between jumps. During each jump the subject wore a belt connected to a Tendo ${ }^{\mathrm{TM}}$ Power Output Unit (Tendo Sports Machines, Trencin, Slovak Republic). The Tendo ${ }^{\mathrm{TM}}$ unit consists of a transducer attached to the end of the belt which measured linear displacement and time. Subsequently, the velocity 
of each jump was calculated and power determined. The average peak and mean power outputs for all five jumps were recorded.

\section{Reaction}

Lower body reaction time was measured with a 20-s reaction test on the Quick Board ${ }^{\mathrm{TM}}$ (The Quick Board, LLC, Memphis, TN) reaction timer. Subjects stood on a board of five circles, in a $2 \times 1 \times 2$ pattern (see Figure 1a). Subjects straddled the middle circle and reacted to a visual stimulus located on a display box that depicted one of five potential lights that corresponded with the circles on the board. Upon activation of the light the subject attempted to move the foot closest to the circle that corresponded to the visual stimulus. Upon a successful connection the next stimulus would appear. The total number of successful attempts for the 20-s test was recorded.

Measurement of hand-eye reaction time was performed on the Dynavision D2 (Dynavision, Ontario Canada). Subjects were required to assume a comfortable athletic stance and stand at a distance from the board where they could easily reach all of the lights (see Figure $1 \mathrm{~b}$ ). The board height was adjusted so the LCD screen was located just below eye level. Participants were told to fixate their gaze on the LCD screen in the middle of the board and to keep their focus there for the entirety of the experiment. During the assessment each subject pressed a light with their dominant side index finger on the board. When a second light flashed (on the same line of the initial light, but on the nondominant side of her body), the subject removed her finger and pressed the new visual stimulus. The time necessary to recognize the new stimulus (new light lit until finger lifted from initial light) was recorded as visual reaction time, and the time it took for the subject to move and press the newly lit light was recorded as the motor reaction time. The total time for both visual reaction and motor reaction was calculated as the physical reaction time. A total of eight attempts were performed. The average time for all eight attempts was recorded.

\section{Player load and heart rate}

All subjects were provided with an individual global positioning system (GPS) that they wore in a vest underneath their playing jersey. The GPS unit (MinimaxX, V4.3, Catapult Innovations, Victoria, Australia) was positioned in a posterior pocket on the vest situated between the subject's right and left scapula in the upper-thoracic spine region. Since the subjects were playing in an indoor facility, there was no viable connection to satellite technology prohibiting information on velocity and distance of activity. However, the ability to
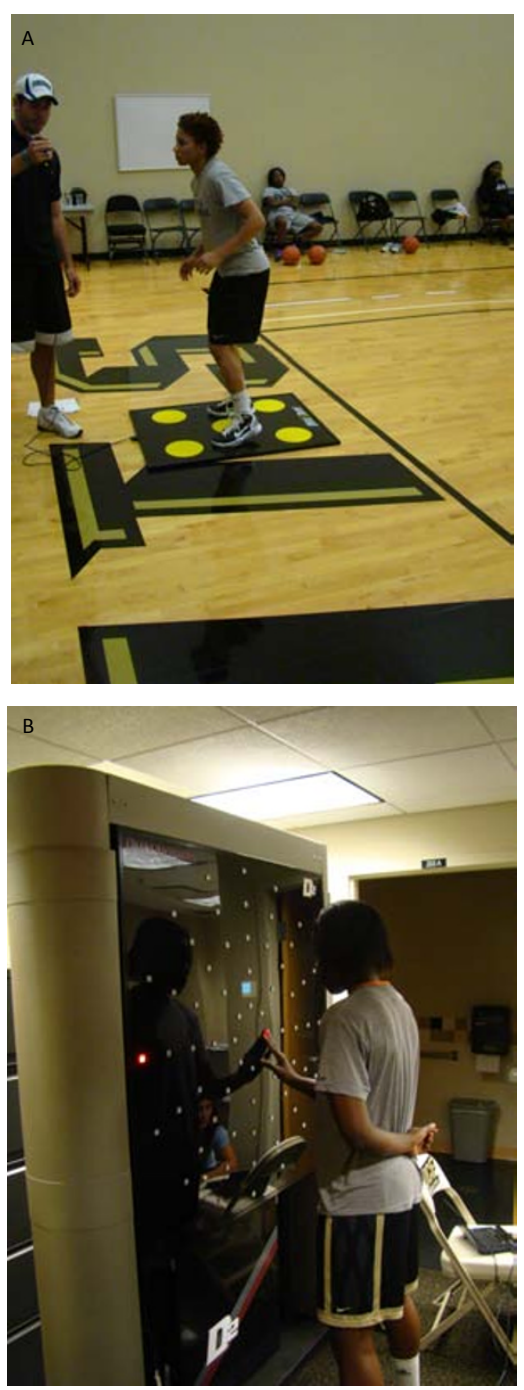

Figure 1 a) Quick Board; B) Dynavision D2

measure all gravitation forces ( $G$ force) in the $G_{Z}, G_{X}$, $G_{Y}$ planes of movement were present. The $G$ forces accumulated during the course of each contest were defined as the Player Load. Player load is an accumulated rate of change of acceleration calculated with the following formula:

Player Load $=\sum_{t=0}^{t=n} \sqrt{\left(\left(f w d_{t=i+1}-f w d_{t=i}\right)^{2}+\left(\text { side }_{t=i+1}-\text { side }_{t=i}\right)^{2}+\left(u_{t=i+1}-u p_{t=i}\right)^{2}\right)}$

Where: Fwd = forward acceleration; side = sideways acceleration; up = upwards acceleration; $\mathrm{i}=$ present time; $\mathrm{t}=$ time.

Data was collected at $10 \mathrm{~Hz}$ and analysis was performed with the system software provided by the manufacturer. The validity and reliability of GPS technology has been demonstrated in several studies [13,14], and specific validity of accelerometry and player load in 
evaluating basketball performance has also been reported [15].

Heart rates were continuously monitored with the Polar FT1 (Polar Electro, Kempele, Finland). Each subject placed the heart rate strap underneath their sports bra. All heart rate data was captured by the GPS unit and downloaded to the GPS computer system following each experimental session.

\section{Basketball shooting performance}

Prior to, and following each game a pre-determined basketball shooting circuit was performed. The circuit required all subjects to shoot 5 balls from 6 different locations on the court (see Figure 2). The total number of successful shots was recorded. The difference between the pregame and post-game shooting performance was calculated and analyzed.

\section{Sweat rate determination, fluid ingestion, and body mass measures}

During the experimental session in which no water was provided subjects were weighed pre and post game. The difference in body mass was attributed to sweat loss. The total body mass loss was used to determine fluid intake in the subsequent experimental sessions. The total fluid loss was recorded and then divided by six. That amount of fluid was provided to each subject at regular intervals. Initial hydration occurred prior to the onset of the game, at minute 10 of the first half, at minute 20 of the first half, prior to the onset of the second half, $10 \mathrm{~min}$ of the second half, and at minute 20 of the second half (at the games conclusion). Subjects were instructed to consume the fluid provided, but were not required to drink the entire amount if they did not feel comfortable. Total water consumed by all subjects was recorded. Body mass was determined $10 \mathrm{~min}$ prior to the warm-up and immediately following post-game data collection.

\section{Statistical analysis}

Since the primary purpose of this investigation was to examine the efficacy of different hydration strategies on the ability to maintain basketball performance, all data assessed prior to and following each game were converted into a $\Delta$ score (Post results - Pre results). All performance data were then analyzed using a one-way repeated measures analysis of variance. In the event of a significant F-ratio, post hoc comparisons using the Fisher's least square difference method was applied to determine pairwise differences. A criterion alpha level of $\mathrm{p} \leq$ 0.05 was used to determine statistical significance.

\section{Results}

The temperature and relative humidity for all games were consistent $\left(22.6 \pm 0.19^{\circ} \mathrm{C}\right.$, and $50.9 \pm 3.1 \%$,

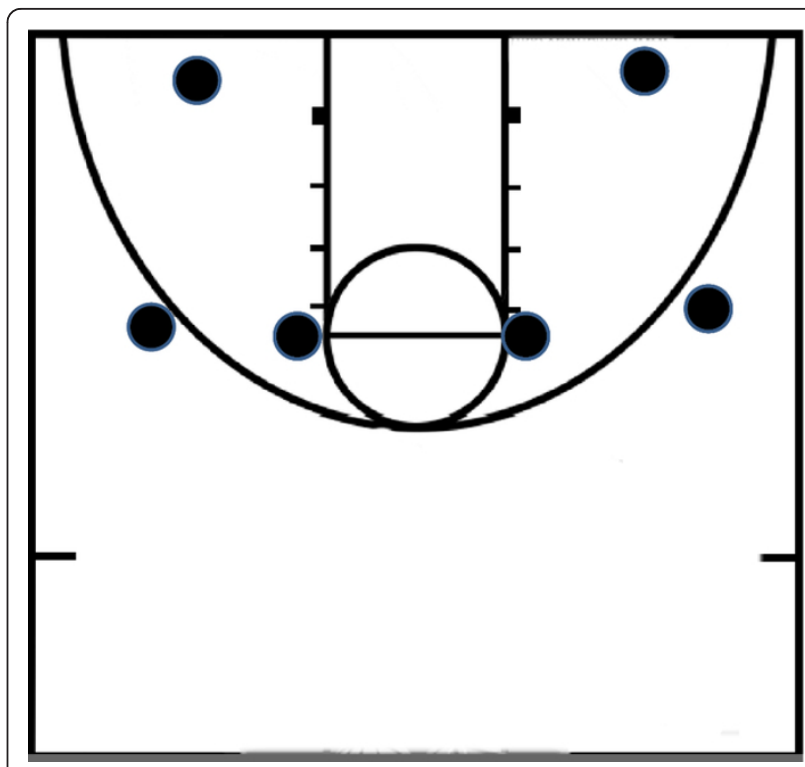

Figure 2 Basketball Shooting Performance

respectively). All subjects began each game in a euhydrated state $\left(\mathrm{U}_{\mathrm{SG}}=1.018 \pm .008\right)$. No significant differences $(p=0.472)$ in $\mathrm{U}_{\mathrm{SG}}$ were seen between trials. During DHY subjects lost $1.72 \pm 0.42 \mathrm{~kg}$, this was equivalent to a $2.3 \%$ loss of their body mass. This was significantly greater than that seen during any other experimental trial (Figure 3). Fluid intake was not significantly different between W, AG1 and AG2 (1.55 \pm $0.43 \mathrm{~L})$.

A significant difference was noted between DHY and AG1 $(p=0.016)$ in the controlled shooting drill (see Figure 4), and a trend was seen between AG2 and DHY $(p=0.094)$. Furthermore, shooting performance was significantly better between AG1 and W $(p=0.029)$.

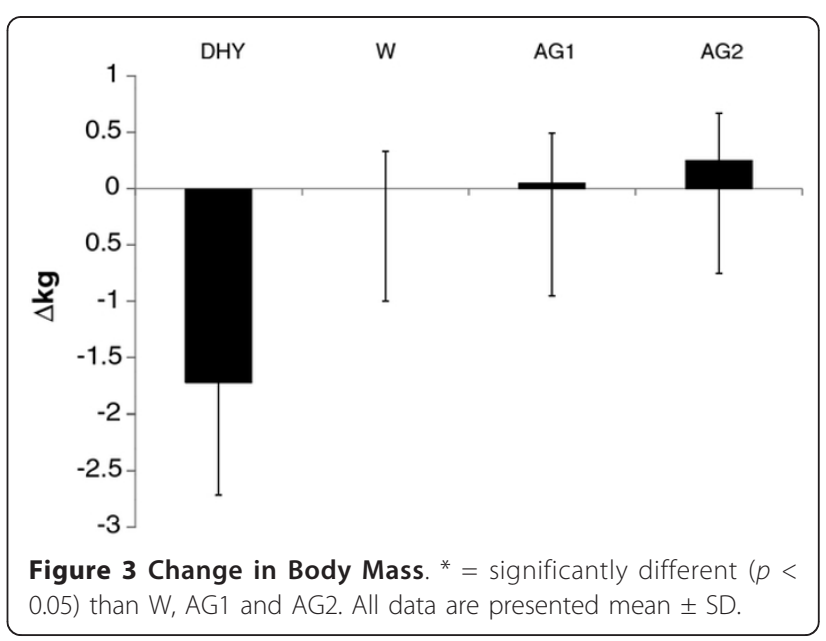


During the AG1 trial subject's shooting percentages were $12.5 \%$ and $11.1 \%$ greater than DHY and $\mathrm{W}$, respectively.

A significant difference in lower body reaction was seen between DHY and the other experimental trials (see Figure 5). No further differences between trials were noted. Visual reaction time (Figure 6a) was significantly better following AG1 $(p=0.014)$ compared to DHY, and a trend toward a similar response $(p=0.081)$ was noted between AG2 and DHY. However no significant differences were noted in the motor response (see Figure $6 \mathrm{~b}$ ). The change in the physical reaction time (combined visual and motor differences) was significantly greater for AG1 compared to DHY $(p=0.032)$.

No significant differences were seen in the pre to post game differences in either peak or mean vertical jump power (see Figures $7 \mathrm{a}$ and $7 \mathrm{~b}$, respectively). Figure 8 depicts the player loads calculated from the GPS device

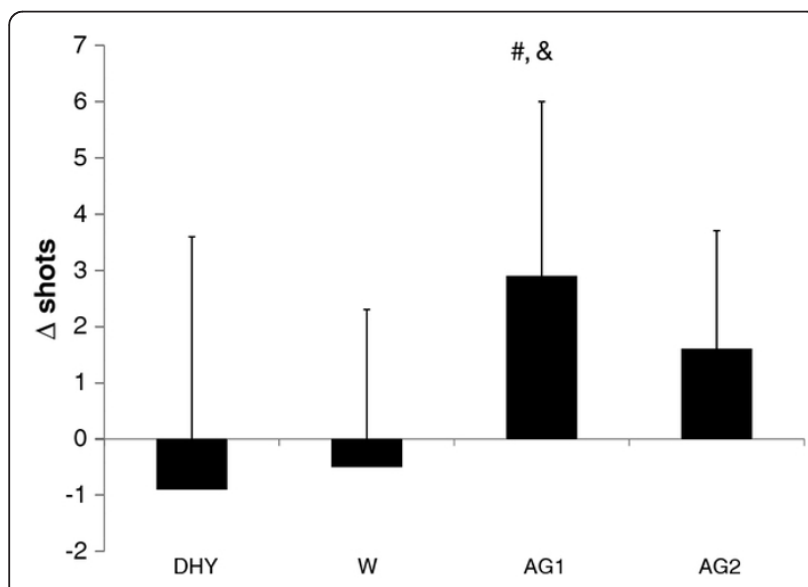

Figure 4 Field Goal Shooting. \# = significantly different than $\mathrm{DHY}$; $\&=$ significantly different than $\mathrm{W}$. All data are presented mean \pm SD.

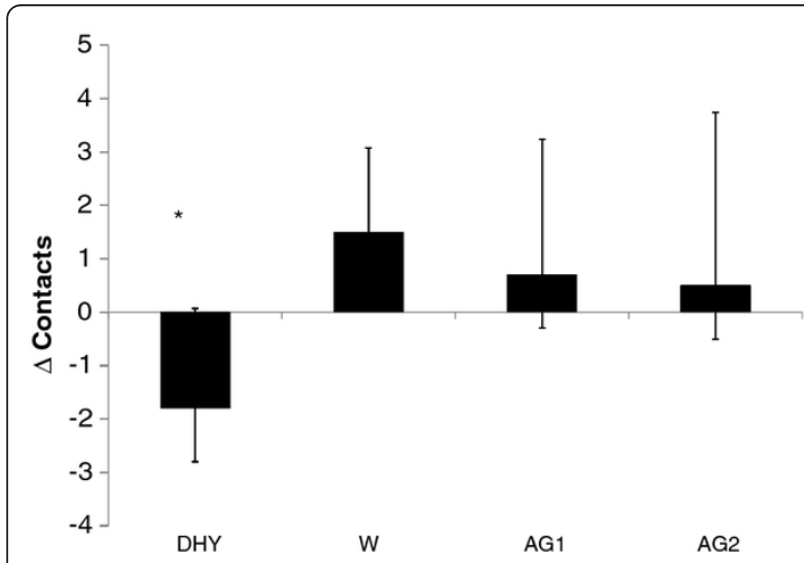

Figure 5 Change in Lower Body Reaction. * significantly different $(p<0.05)$ than $W, A G 1$ and AG2. All data are presented mean \pm SD

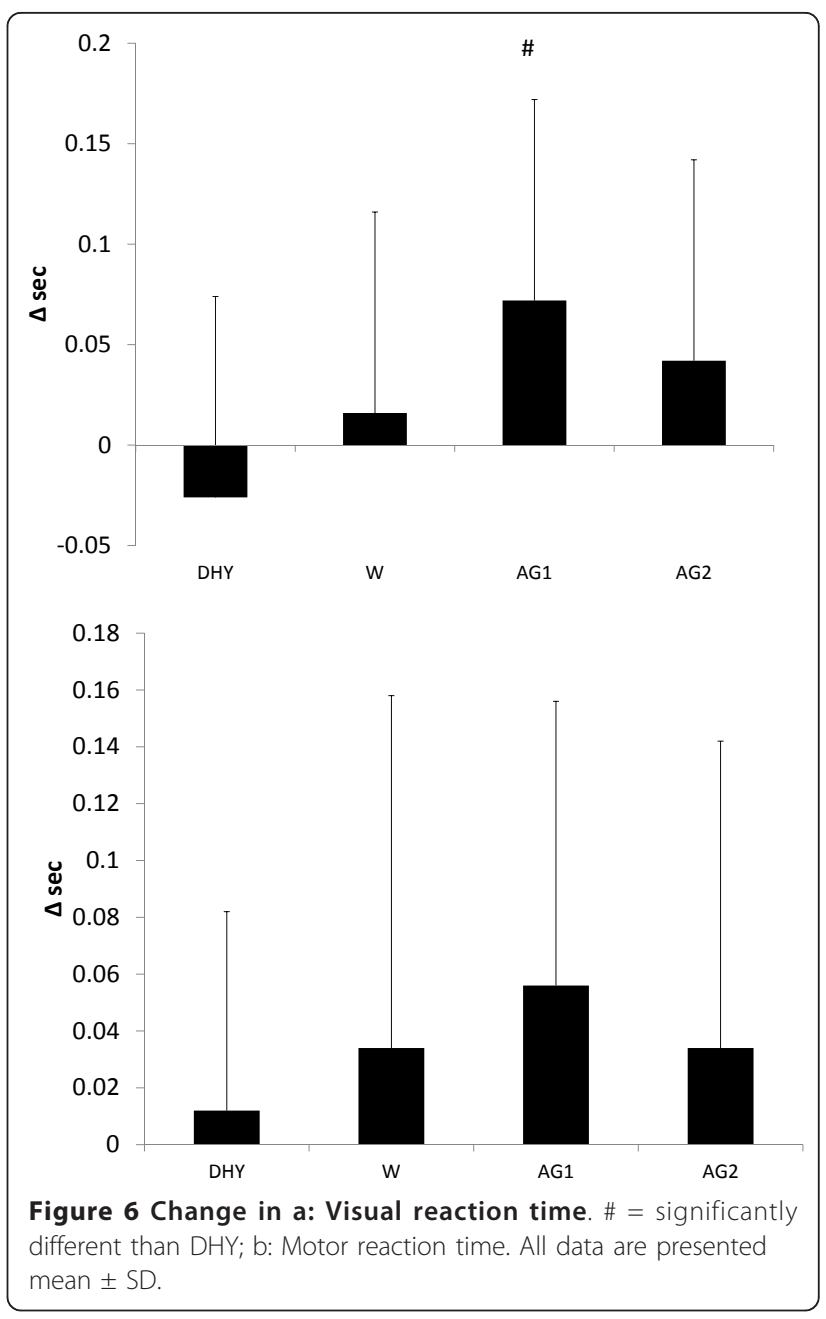

during each game. During AG2 a significantly greater player load was seen compared to DHY $(p=0.045)$. A trend for greater player loads were also noted between AG1 $(p=0.064)$ and W (0.073) compared to DHY. Average heart rates during each experimental trial are depicted in Table 1. No significant differences were noted in average heart rate between each trial. Although heart rates were $4.5 \%$ to $5.3 \%$ lower in all trials compared to DHY, these differences were not statistically different.

\section{Discussion}

Results of this study indicate that female basketball players lose approximately $2.3 \%$ of their body mass during a game in which they are not permitted to rehydrate. Despite a significant loss of body fluid during DHY subjects were able to maintain jump power throughout the game, but basketball shooting performance and reaction time was significantly impaired. Rehydration trials using AG was able to maintain basketball shooting accuracy to a better extent than water 

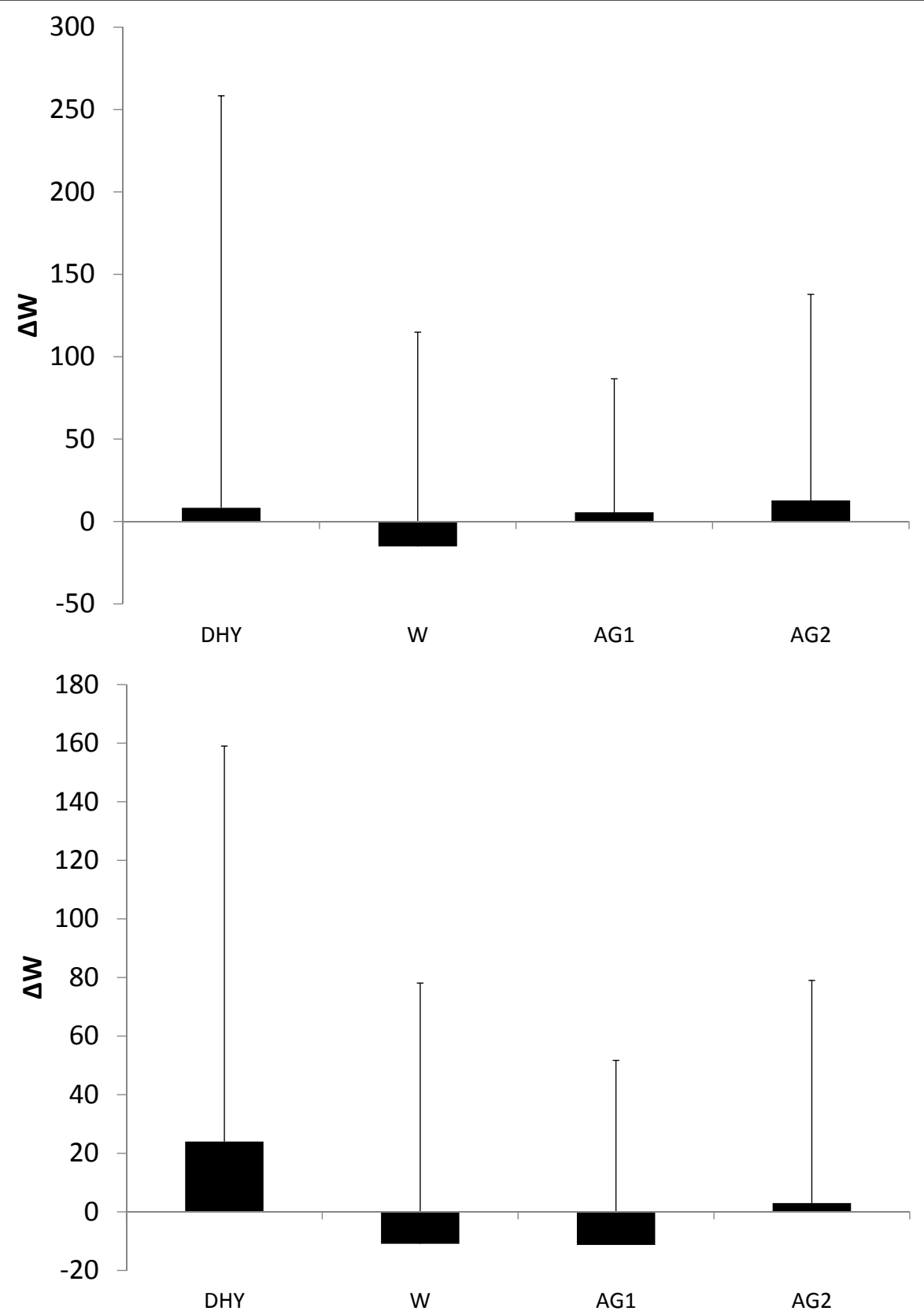

Figure 7 Change in: $\mathbf{a}=$ Peak Vertical Jump Power; $\mathbf{b}=$ Mean Vertical Jump Power. All data are presented mean \pm SD.

alone, and ingestion of AG1 also enhanced visual reaction time. Subjects consuming the supplement were able to respond to a visual stimulus quicker than when dehydrated. No significant differences in visual reaction time were observed in subjects ingesting water compared to the dehydrated condition. Lower body reaction time was significantly reduced when subjects were not permitted to rehydrate, however no differences were seen between water and AG ingestion.
The level of hypohydration seen in this study was similar $(2.3 \%$ versus $2.0 \%)$ to previous research examining a 40-min basketball game in men [9]. The effect of this mild hypohydration stress on jump power performance was consistent with previous research examining the effect of mild to moderate levels of hypohydration on jump or repetitive jump performance $[9,16,17]$. Judelson and colleagues [17] showed that jump power is maintained following dehydration protocols that elicited 


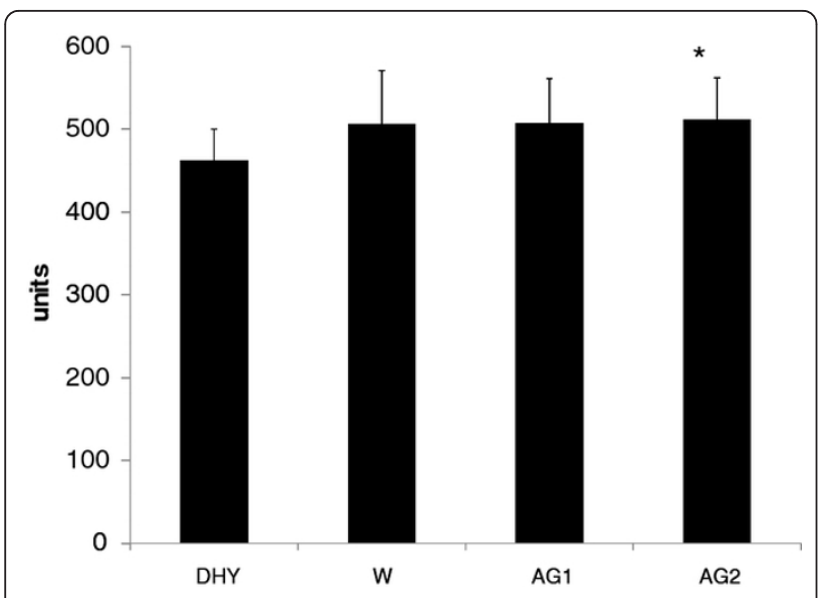

Figure 8 Player Load. \# = significantly different than DHY. All data are presented mean \pm SD.

a $2.5 \%$ and a $5.0 \%$ loss of body mass. Similarly, Cheuvront et al., [16] also reported no decrement in jump power performance in men following a $3.8 \%$ loss in body mass. Taken together, it appears that mild to moderate levels of dehydration do not impair jumping performance

Dehydration has been shown to impair basketball shooting performance in several studies $[8,9,18]$. While Hoffman and colleagues [9] reported that a $2 \%$ level of dehydration can decrease shooting percentages by $8 \%$ (results not statistically different), others have shown that a similar level of hypohydration can cause significant performance decrements in shooting accuracy [18] and that it can progressively decline with greater levels of fluid loss [8]. The results of this present investigation are consistent with these latter studies. The mechanism that may have contributed to a decrease in shooting percentage may be fatigue relating to the hydration stress. However, considering that power outputs remained consistent between experimental trials and no difference in player load was observed between DHY and AG1, it is more likely that other factors contributed to the differences observed in shooting percentages between DHY and AG trials.

A recent investigation has indicated that moderate levels of dehydration ( $4 \%$ body mass loss) can result in

Table 1 Average Heart Rates

\begin{tabular}{cccc}
\hline & First Half & Second Half & Entire Game \\
\hline DHY & $176.8 \pm 8.2$ & $174.5 \pm 7.5$ & $175.7 \pm 7.3$ \\
\hline $\mathbf{W}$ & $169.2 \pm 9.9$ & $164.6 \pm 15.9$ & $166.8 \pm 10.8$ \\
\hline AG1 & $167.7 \pm 13.4$ & $168.5 \pm 9.7$ & $168.1 \pm 11.2$ \\
\hline AG2 & $166.9 \pm 11.9$ & $166.5 \pm 13.3$ & $166.7 \pm 12.3$ \\
\hline P value & 0.186 & 0.286 & 0.200 \\
\hline All data are presented as mean \pm SD & &
\end{tabular}

significant alterations in afferent neural processing [19]. This suggests that the ability to maintain fine motor control in performance, such as shooting a basketball, may become significantly impaired during a hypohydration stress. Additional research has also indicated that dehydration can increase lateral ventricle enlargement in the brain causing a higher level of neuronal activity in the brain required to achieve the same performance level $[20,21]$. This may explain in part the significant performance decrements observed in reaction time (both visual and in lower body) during DHY. When subjects were permitted to rehydrate (regardless of $\mathrm{W}$ or AG) lower body reaction times were significantly improved. However, the ingestion of AG1 significantly enhanced basketball shooting performance to a greater extent $(p<0.05)$ than W only. In addition, AG1 improved visual reaction time during the competition, whereas no difference was observed between $\mathrm{W}$ and DHY. Although not statistically different, similar trends were seen between AG2 and shooting accuracy and visual reaction time ( $p=0.09$ and $p=0.08$, respectively). The ability to enhance visual reaction time with AG1 does appear to have important implication for athletic performance. Mann and colleagues [22] have suggested the ability to process visual information provides critical information for enhancing the anticipatory response during athletic performance. Achieving excellence in basketball has been suggested to be related in part to an ability of the athlete to have a "highly-tuned" anticipatory ability that allows them to predict other's actions ahead of their realization [23].

Rehydrating with AG during the rest breaks of the game may have contributed to a more efficient fluid and electrolyte uptake, minimizing the deleterious effects of dehydration. Previous research has demonstrated that the AG dipeptide can enhance fluid and electrolyte absorption in the gut, especially at a low $\mathrm{pH}$, which is common during exercise $[5,6]$, and that when consumed by subjects during a mild hydration stress $(-2.5 \%$ of body mass loss) exercise can be prolonged to a greater extent than with water ingestion only [7]. Although speculative, AG ingestion may have augmented fluid uptake from the gut, and minimized the potential deleterious effects that mild levels of dehydration had on nerve conduction and brain function. These effects may be more prevalent in activities involving multisensory information such as shooting (involves a coordinated and precise visual and motor control of the hands and arms) versus reaction of the lower body.

In conclusion, rehydration with AG appears to maintain basketball skill performance and visual reaction time to a greater extent than water only. These effects are likely mediated by enhanced fluid and electrolyte uptake from the gut and subsequent preservation of 
neural function that commands physical activities involving fine motor control. Further research appears warranted in the examination of AG ingestion and neural activity during periods of hydration stress.

\section{Acknowledgements}

The authors would like to thank a dedicated group of subjects. This study was supported by a grant from Kyowa Hakko USA, New York, NY.

\section{Authors' contributions}

JRH was the primary investigator, designed study, supervised all study recruitment, data/specimen analysis, statistical analysis and manuscript preparation. DRW, NSE, MWH, AJW, DMN, WPM, GTM and AMG were coauthors, assisting with data collection and data analysis. MSF helped drafting the drafting the manuscript. All authors read and approved the final manuscript.

\section{Competing interests}

The authors declare that they have no competing interests.

Received: 15 January 2012 Accepted: 7 March 2012

Published: 7 March 2012

\section{References}

1. Nath SK, Dechelotte P, Darmaun D, Gotteland M, Rongier M, Desjeux JF: $\left({ }^{15} \mathrm{~N}\right)$ and $\left({ }^{14} \mathrm{C}\right)$ glutamine fluxes across rabbit ileum in experimental diarrhea. Am J Physiol 1992, 262:G312-G318.

2. Silva AC, Santos-Neto MS, Soares AM, Fonteles MC, Guerrant RL, Lima AA: Efficacy of a glutamine-based oral rehydration solution on the electrolyte and water absorption in a rabbit model of secretory diarrhea induced by cholera toxin. J Pediatr Gastroenterol Nutr 1998, 26:513-519.

3. van Loon FP, Banik AK, Nath SK, Patra FC, Wahed MA, Darmaun D, Desjeux JF, Mahalanabis D: The effect of L-glutamine on salt and water absorption: a jejuna perfusion study in cholera in humans. Eur $\mathrm{J}$ Gastroenterol Hepatol 1996, 8:443-448.

4. $L i$ Y, Xu B, Liu F, Tan L, Li J: The effect of glutamine-supplemented total parenteral nutrition on nutrition and intestinal absorptive function in a rat model. Pediatr Surg Int 2006, 22:508-513.

5. Lima AA, Carvalho GH, Figueiredo AA, Gifoni AR, Soares AM, Silva EA, Guerrant RL: Effects of an alanyl-glutamine-based oral rehydration and nutrition therapy solution on electrolyte and water absorbtion in a rat model of secretory diarrhea induced by cholera toxin. Nutr 2002, 18:458-462.

6. Fürst P: New developments in glutamine delivery. J Nutr 2001, 131(suppl):2562-2568.

7. Hoffman JR, Ratamess NA, Kang J, Rashti SL, Kelly N, Gonzalez AM, Stec M, Andersen S, Bailey BL, Yamamoto LM, Hom LL, Kupchak BR, Faigenbaum AD, Maresh CM: Examination of the efficacy of acute LAlanyl-L-Glutamine during Hydration Stress in Endurance Exercise. J Int Soc Sports Nutr 2010, 7:8.

8. Dougherty KA, Baker LB, Chow M, Kenney WL: Two percent dehydration impairs and six percent carbohydrate drink improves boys basketball skills. Med Sci Sports Exerc 2006, 38:1650-1658.

9. Hoffman JR, Stavsky H, Falk B: The effect of water restriction on anaerobic power and vertical jumping height in basketball players. Int I Sports Med 1995, 16:214-218.

10. Rothstein A, Adolph EF, Wells JH: Voluntary dehydration. In Physiology of Man in the Desert. Edited by: Adolph EF. New York: Interscience: 1947:254-270.

11. Osterberg KL, Horswil CA, Baker LB: Pregame urine specific gravity and fluid intake by National Basketball Association players during competition. J Athl Train 2009, 44:53-57.

12. Armstrong LE, Maresh CM, Castellani JW, Bergeron MF, Kenefick RW, LaGasse KE, Riebe D: Urinary indices of hydration status. Int I Sport Nutr 1994, 4:265-279.

13. Coutts AJ, Duffield R: Validity and reliability of GPS devices for measuring movement demands of team sports. J Sci Med Sport 2010, 13:133-135.
14. Gray AJ, Jenkins D, Andrews MH, Taaffe DR, Glover ML: Validity and reliability of GPS for measuring distance travelled in field-based team sports. J Sport Sci 2010, 28:1319-1325.

15. Montgomery PG, Pyne DB, Minahan CL: The physical and physiological demands of basketball training and competition. Int I Sport Physiol Perf 2010, 5:75-86.

16. Cheuvront SN, Kenefick RW, Ely BR, Harman EA, Castellani JW, Frykman PN, Nindl BC, Sawka MN: Hypohydration reduces vertical ground reaction impulse but not jump height. Eur J Appl Physiol 2010, 109:1163-1170.

17. Judelson DA, Maresh CM, Farrell MJ, Yamamoto LM, Armstrong LA, Kraemer WJ, Volek JS, Speiring BA, Casa DJ, Anderson JM: Effect of hydration state on strength, power, and resistance exercise performance. Med Sci Sports Exerc 2007, 39:1817-1824.

18. Baker LB, Kougherty KA, Chow M, Kenney WL: Progressive dehydration causes a progressive decline in basketball skill performance. Med Sci Sports Exerc 2007, 39:1114-1123.

19. Montain SJ, Tharion WJ: Hypohydration and muscular fatigue of the thumb alter median nerve somatosensory evoked potentials. Appl Physiol Nutr Metab 2010, 35:456-463.

20. Kempton MJ, Ettinger U, Foster R, Williams SC, Calvert GA, Hampshire A, Zelaya FO, O'Gorman RL, McMorris T, Owen AM, Smith MS: Dehydration affects brain structure and function in healthy adolescents. Hum Brain Mapp 2011, 32:71-79.

21. Kempton MJ, Ettinger $U$, Schmechtig A, Winter EM, Smith L, McMorris T, Wilkinson T, Williams SC, Smith MS: Effects of acute dehydration on brain morphology in health humans. Hum Brain Mapp 2009, 30:291-298.

22. Mann DL, Abernathy B, Farrow D: Visual information underpinning skilled anticipation: The effect of blur on a coupled and uncoupled in situ anticipatory response. Atten Percept Psychophys 2010, 72:1317-1326.

23. Aglioti SM, Cesari P, Romani M, Urgesi C: Action anticipation and motor resonance in elite basketball players. Nat Neurosci 2008, 11:1109-1116.

doi:10.1186/1550-2783-9-4

Cite this article as: Hoffman et al.: L-alanyl-L-glutamine ingestion maintains performance during a competitive basketball game. Journal of the International Society of Sports Nutrition 2012 9:4.

\section{Submit your next manuscript to BioMed Central and take full advantage of:}

- Convenient online submission

- Thorough peer review

- No space constraints or color figure charges

- Immediate publication on acceptance

- Inclusion in PubMed, CAS, Scopus and Google Scholar

- Research which is freely available for redistribution

Submit your manuscript at www.biomedcentral.com/submit
Ciomed Central 